\title{
SELF-ASSEMBLED ROV AND PHOTOGRAMMETRIC SURVEYS WITH LOW COST TECHNIQUES
}

\author{
E. Costa ${ }^{\text {a }}$, F. Guerra ${ }^{\text {a }}$, P. Vernier ${ }^{\text {a }}$ \\ a Università Iuav di Venezia, S. Croce 191, 30135 Venezia, Italy - (ecosta, guerra2, vernier)@iuav.it)
}

Commission II, WG II/9

KEY WORDS: Remotely Operated Vehicle, Underwater Photogrammetry, Point clouds, Survey digital techniques

\begin{abstract}
:
In last years, ROVs, have been employed to explore underwater environments and have played an important role for documentation and surveys in different fields of scientific application. In 2017, the Laboratorio di Fotogrammetria of Iuav University of Venice has decided to buy an OpenRov, a low cost ROV that could be assembled by ourselves to add some external components for our necessities, to document archaeological sites.

The paper is related to the photogrammetric survey for the documentation of underwater environments and to the comparison between different solutions applied on a case studio, five marble columns on a sandy bottom at 5 meters deep. On the lateral sides of the ROV, we have applied two GoPro Hero4 Session, which have documented the items both with a series of images and with a video. The geometric accuracy of the obtained 3D model has been evaluated through comparison with a photogrammetric model realized with a professional reflex camera, Nikon D610. Some targets have been topographically surveyed with a trilateration and have been used to connected in the same reference system the different models, allowing the comparisons of the point clouds.

Remote Operating Vehicles offer not only safety for their operators, but are also a relatively low cost alternative. The employment of a low-cost vehicle adapted to the necessities of surveys support a request for safer, cheaper and efficient methods for exploring underwater environments.
\end{abstract}

\section{INTRODUCTION}

ROVs, called also UROVs, Underwater Remotely Operated Vehicle, have been extensively used by researchers to explore underwater environments, both in shallow and deep water, for different kind of studies, as marine science and underwater archaeology, playing an important role for documentation and surveys (Bruno et al., 2015; Nornes at al., 2015; Ødegård et al., 2016). The most innovative technologies in recent years have led to improve the quality of underwater surveys; the use of ROVs supports the work of archaeologists, especially in those contexts of difficult accessibility, as wrecks at greater depths (Scaradozzi et al., 2013). Also at depths where divers can work easily but with reduced dive time, the ROVs, assembled with cameras, could realize a photogrammetric survey in a single dive, due to the battery autonomy around 3 hours.

Usually, the operation of drawing and representation of an archaeological site requires an considerable effort of and economic resource. The possibilities to add some tools for underwater documentation as cameras applied on ROV, without the employment of divers or in addition to them, could be very advantageous also on reasonable depths, but that could requires professional divers.

Our ongoing study aims to provide information to researchers, archaeologists or biologists, which work in an underwater environment and which could obtain information by means of documentation and survey of cultural heritage.

The aims of our research starts from the necessity to understand the advantages and the limitations of the use of a little and lowcost ROV, comparing it with the experties and knowledge acquired by the authors and by archaeological divers in underwater survey in the last 10 years. What are the advantages and disadvantages of a small ROV? What could add to the quality of a survey realized by a diver?

\section{THE ROV}

'A primary interest of the archaeological investigation is to extract, in a non-invasive way, as much information as possible with minimal expenditure of time and of expensive resources' (Scaradozzi et al., 2013). Traditionally, ROVs are high value devices and therefore of limited access for small researchers groups, but, recently, many industries are producing low-cost ROVs that could be afforded by a great public (Teague et al., 2017). Between many possibilities, the Laboratorio di Fotogrammetria has decided to buy the OpenRov, an opensource and a very low-cost ROV that could be assembled independently and could be freely modified for custom uses (Heisinger et al., 2017) .

It is very little and light, but, at the same time, could reach 100 $\mathrm{m}$ of depth. The external structure is laser cut from acrylic panels and contains a transparent cylinder for the internal HD webcam, LED lights and the electronic parts. These are connected to a series of Lithium batteries, sealed inside two lateral transparent tubes, which give power and stability to the vehicle. Three engines compose the electronic components related to propulsion: two horizontal for lateral movements and one vertical for buoyancy and depth. An IMU platform (Inertial Measurement Unit) has been applied to enable and control highly accurate depth, compass heading, roll and pitch. The ROV is controlled through an Ethernet cable connected to a

* Corresponding author 
laptop with a gamepad, to give the possibility to easily drive the vehicle directly from the boat or from the shore (Figure 1).

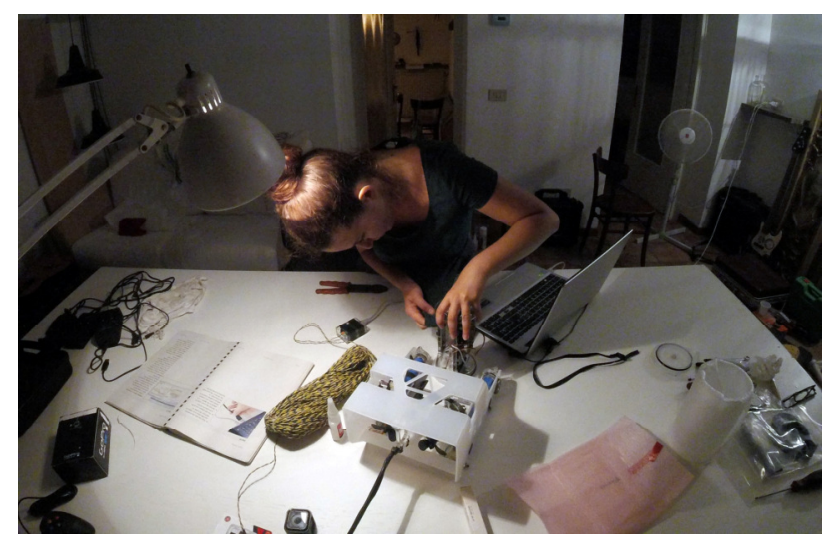

Figure 1. One of the phases of assembling the ROV.

The OpenRov have an inner camera used only to drive and control the vehicle, so we have decided to tighten, on the lateral sides of the structure, two cameras with their underwater housings. The employment of the GoPro Hero4 Session (Figure

2 ), a recreational camera, is mainly connected to its low cost and little dimension, but also to the neutral weight of this camera underwater, which not compromise the buoyancy of the ROV. The buoyancy has been checked before the beginning of the survey (Figure 3). The use of a self-assembled rov has permitted to modify some components, such as two little weights on the bow, which has been moved to the stern to allow the correct movement forward, since the two lateral wings created by the two cameras have modified the longitudinal buoyancy. With these cameras, the authors have been the possibilities to record both images and HD videos, to perform photogrammetric surveys, to build a photomosaic and a textured 3D model.

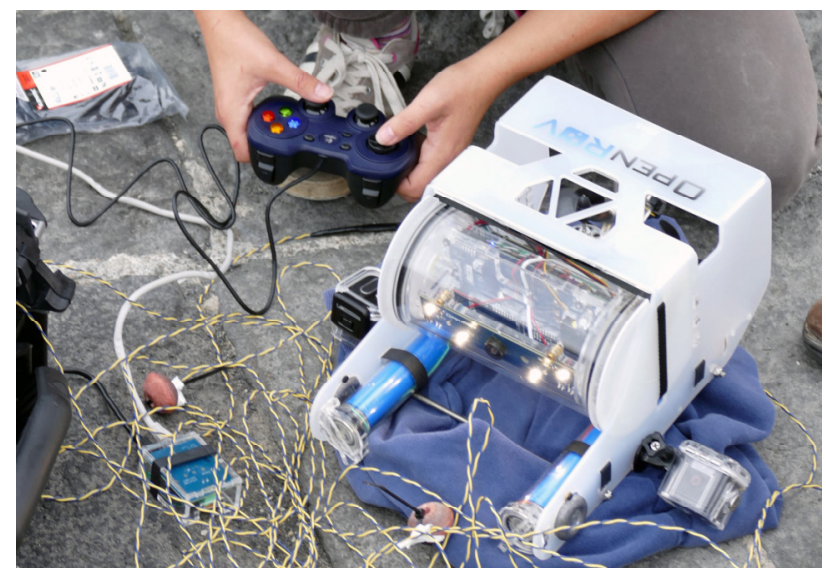

Figure 2. The OpenRov with the two GoPro mounted on sides.
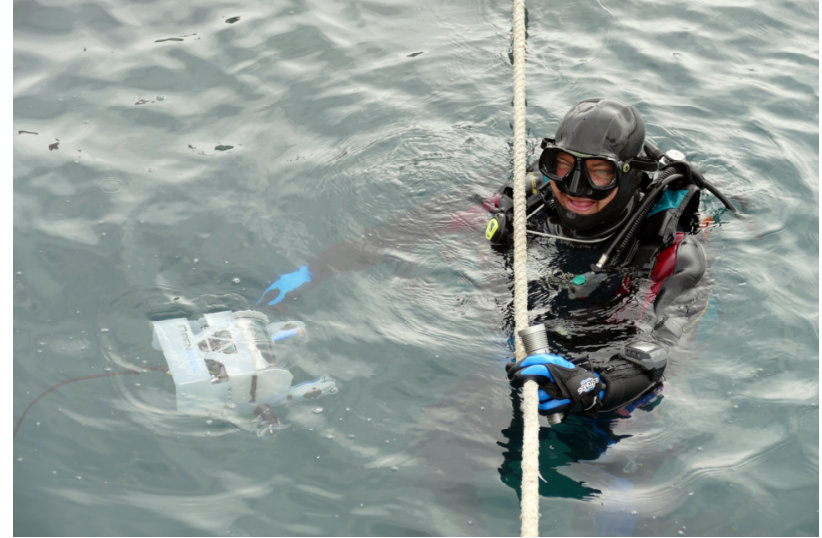

Figure 3. One of test on the buoyancy inside the harbour.

\section{THE DOCUMENTATION}

These technologies have been utilized for mapping and monitoring the cultural and natural heritage or to document the phases of the work of the archaeologist during the excavation, but the principal application of the ROVs is related to photogrammetric survey for the documentation of underwater environments (Drap et al., 2015; Sedlazeck et al., 2009, Teague et al., 2017).

To test the ROV and apply the photogrammetric survey in an underwater contest, it has been decided to investigate an easy and accessible wreck; near Torre Chianca, in Puglia, Italy, at around one hundred meters from the coast, five columns lays on a sandy bottom at 5 meters deep (Figure 4).

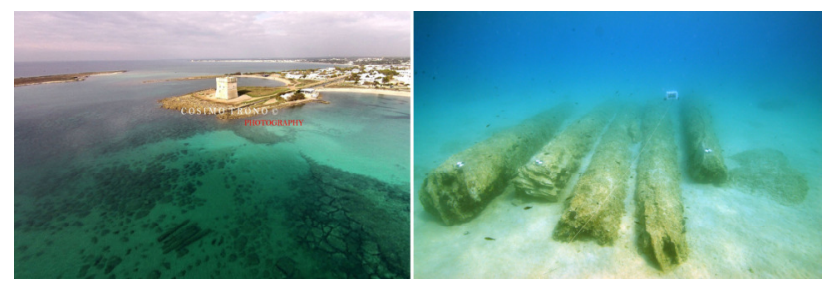

Figure 4. The five columns of the archaeological site. (On the left, photo: Cosimo Tronto. http://www.leccesette.it/ dettaglio.asp?id_dett=23429\&id_rub=113)

First of all, $6 \mathrm{~B} / \mathrm{W}$ targets have been initially positioned on the items and have been measured with a trilateration survey in order to obtain $3 \mathrm{D}$ coordinates, to georeferenced the items in a local reference system and the control the metrical accuracy of the photogrammetric models, checking the $\mathrm{x} y \mathrm{z}$ coordinates of the ground control points. (Figure 5).

Photogrammetrical surveys have been realized both by the diver with nadiral and radial strips around and between the columns, both by the cameras of the ROV, only with nadiral strips. 


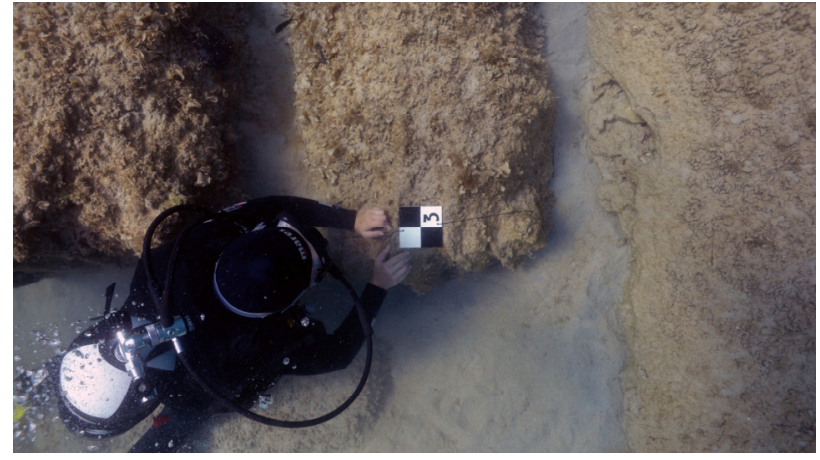

Figure 5. A B/W target surveyed with trilateration.

\subsection{Multi-image photogrammetry}

Three different virtual models have been obtained:

- with the images taken by Nikon D610

- with the images taken by GoPro Hero4 Session

- with the video taken GoPro Hero4 Session

The photogrammetric block with the reflex camera has been manually realized by the diver, obtaining around 400 images with $6016 \times 4016$ pixels and a resolution of 300 dpi, subdivided in parallel and regular strips with an overlap between the images around $60 \%$ and between the strips around $20 \%$ (Figure $6)$.

The GoPro camera has acquired images of medium dimension, 2720x 2040 pixels, and with a resolution of 72 dpi), and has been setted automatically to record an image every 0.5 seconds to ensure maximum overlap between images (Figure 7). The driver don't have the possibilities to set the route of the fly, but he has to manually drive the vehicle. On the OpenROV we have attached an IMU platform, that control the orientation to the Nord of the vehicle, the roll and the pitch and the depth, but can not calculate the position in the space; this sensor is widely used in underwater vehicles due to its low cost, this systems is less precise than the expensive ones, generating significant errors (Martínez et al., 2013).

Consequently, the strips of the ROV are less regular and parallel than those of the diver, due to the difficulties to maintain the correct lines in the underwater current. Furthermore, in this first test it has been noticed that the tether cable had a negative buoyancy and it remained stuck on the edge and the concretions of the columns, stopping the parallel movement of the ROV. In following tests, we have organized the cable with some positive buoy at $5 \mathrm{~m}$, which took the cable in neutral position.

The use of the stereo camera, with a known distance of the central point on the lenses of the objectives, (Negahdaripour et al., 2005, Schmidt et al., 2012) permits to obtain a scaled photogrammetric model. This technique is useful when, working at high depths, it is not possible to place targets on the subject and realize a topographical survey, or during monitoring and survey campaigns.

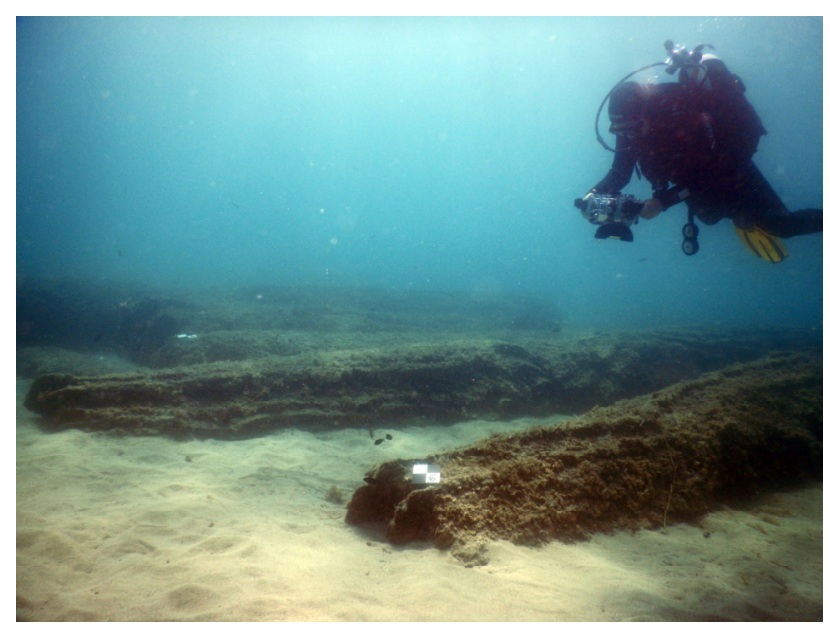

Figure 6 . The imaging capture during the photogrammetric survey.

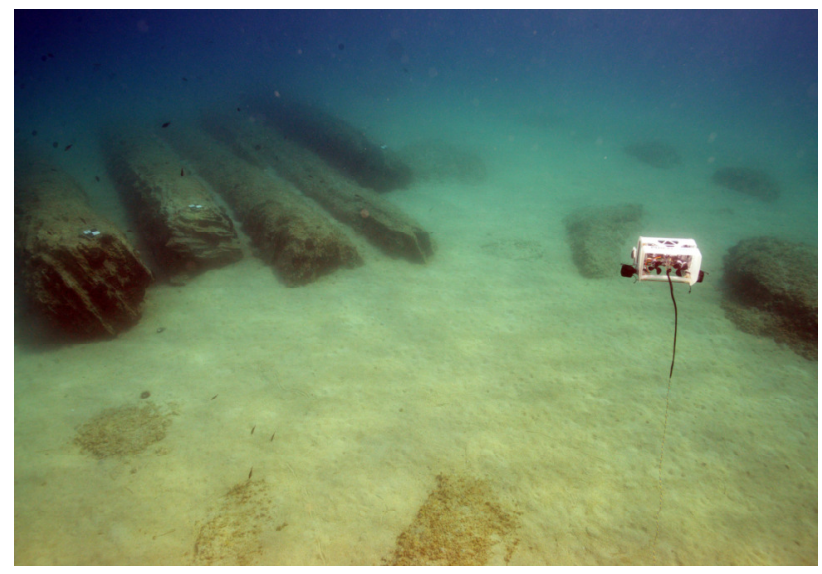

Figure 7. The ROV and the columns of the shipwreck.

\subsection{The comparison between point clouds}

The comparison between the point clouds has been realized by software CloudCompare. Point clouds produced by photogrammetric survey were fitted together to show the difference between them. The point clouds have been oriented in the same reference system with the 6 targets (GCP) placed on the columns. The photogrammetric software employed for the alignment of the images is the same for every project, so the algorithm employed has been the same, to obtain the same raytracing, and the settings of the alignment and the creation of the dense clouds are the same. The comparison has given good results with a high conformity between the points, whit a mean error and an standard deviation $<1 \mathrm{~cm}$, below our expectations, considering the lack of a good process of calibration of the cameras in an underwater environment. The range of the scale has been set from 0 to $3 \mathrm{~cm}$ and the colour changes from blue to red; in this software it is not possible realized the comparison with the signed-distance, but for the analysis that we want to obtain, it is not necessary, but it is important to know that there is or not a difference.

\subsubsection{Comparison between images obtained by GoPro and by Nikon}

The first comparison were performed on the point cloud coming from photogrammetric survey with the images obtained with the 
Nikon D610 and GoPro Hero 5 Session. We have found obtained around $1.1 \mathrm{~cm}$, show in green in the followings model (Figure 8). Observing the Gauss curve of the standard deviations and the histogram of the absolute distances between the points in figure 9, the average is calculated on $0.0015 \mathrm{~m}$, with a majority of blue colour points.

Just because most of the part is in blue and some little portions in green, it's clear that the two models fit and little differences are due to the photogrammetric block and the different distortions of the lenses of the camera, due to the absence of a on site calibration.

The external red parts in the comparison do not represent an error, but an absence of the data around the colums because the area documented with the two cameras is different; that one in the middle of the columns is relative at the absence of the radial photogrammetrical images on the survey realized with the ROV.

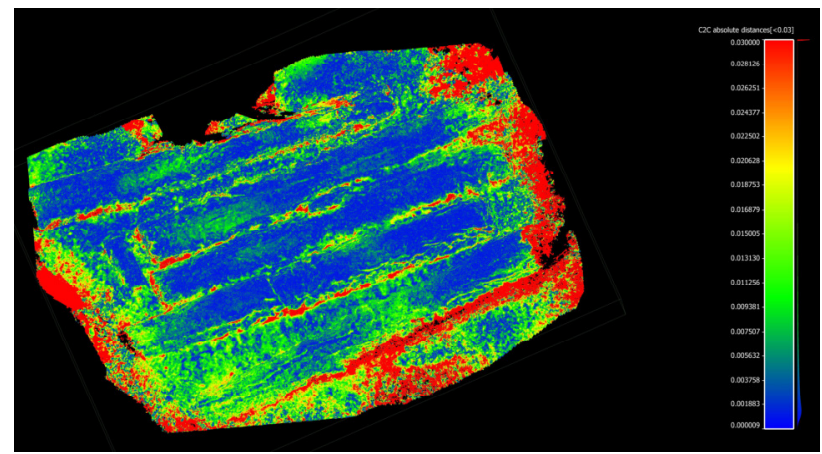

Figure 8. Point clouds of GoPro photogrammetric survey. Compared distance with points cloud obtained with Nikon D610.
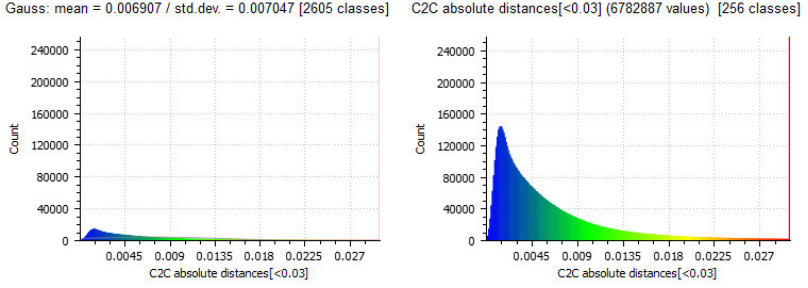

Figure 9. Statistical analysis with Gauss Curve and Histogram on the Photogrammetric point cloud of GoPro - Nikon.

The figure 10, which represents the same point clouds from two different views, shows the comparison between the complete photogrammetric images of the Nikon D610 and the model realized only with nadiral images, to highlight how the radial images are necessary to obtain a complete model.

Analyzing this result, in order to make an accurate survey with the ROV, the researchers have to set up two different photogrammetric session:

- the first survey with the cameras set nadirally to the subject

- the second one with the cameras rotated and positioned at 45 , to better represent the lateral portion of the columns.

Contrary to nadiral strips in which cameras can be triggered simultaneously to realized stereo pairs of images, the stereoscopic orientation is lost with radial strips with the camera at $45^{\circ}$. The rov can not swim sideways, therefore the camera can not be positioned at $45^{\circ}$ with forward orientation but only laterally.

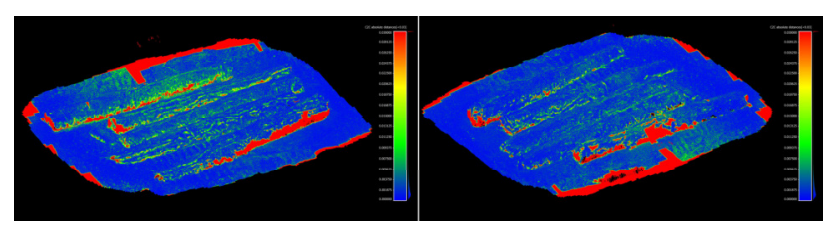

Figure 10. Point clouds of Nikon nadiral image photogrammetric survey. Compared distance with points cloud obtained by Nikon radial images.

\subsubsection{Comparison between images and videos obtained by GoPro}

The second comparison has been made between the two photogrammetric models obtained with the images and with the video realized by GoPro Hero4 Session. A first difference could be noticed directly on figure 11: the RGB value and the dimension of the images are different. The two picture at the top show the original images of the GoPro, with a dimension of $2720 \times 2040$ pixels and are characterized by a green dominant colour, while the two picture on the bottom of the figure represent the frames extrapolated from the video, which have, instead, a different dimension of $1920 \times 1080$ pixels and are characterized by a blue dominant colour. Automatically adjusts of the color tone based on the environmental conditions of the GoPro works on different value of the white balance.
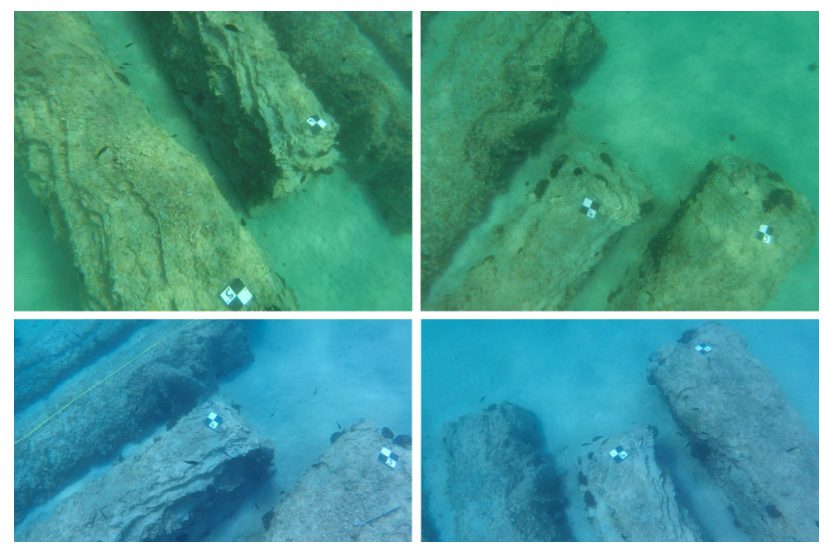

Figure 11. On the top, images of GoPro. On the bottom, the same subject on two frames from the video. Underwater, the typical radial distortion of fish-eye lenses disappear from the images.

The photogrammetric process of the video has been processed by the Photoscan software in the latest version 1.4.0 which is able to extract the frames from the video. The videos of the ROV concerning the photogrammetric strips last 12 minutes each; selecting as the setting a frame step 20, it is possible to obtain a frame every 0.62 seconds, for a total of 2160 images, reduced to 1390 after a careful clearing. The images obtained with the GoPro were about the same number; in fact, setting of the photoshooting was a frame every 0.5 seconds. The realization of the photogrammetric survey obtained with the frames extrapolated from the video was more complicated; the alignment of the frames has not been complete on a column, but a hundred of not aligned images ha sto be realigned in a second chunk; only in a second time, the differnt chinks have been merged together, applying control points directly on the edge of the columns. The comparison between the point clouds of the images and the video was not as precise as the previous one. IN 
the center of the model the software has calculate a greater error $(0.03 \mathrm{~m})$. Positioning a GCP in the center of the model and comparing the errors obtained on the coordinates $x \mathrm{y} z$, the error results on the $\mathrm{z}$, confirming a difference on the distance between the poin clouds only in the depth. The model obtained with the video have a central distortion that it is absent on the other models.

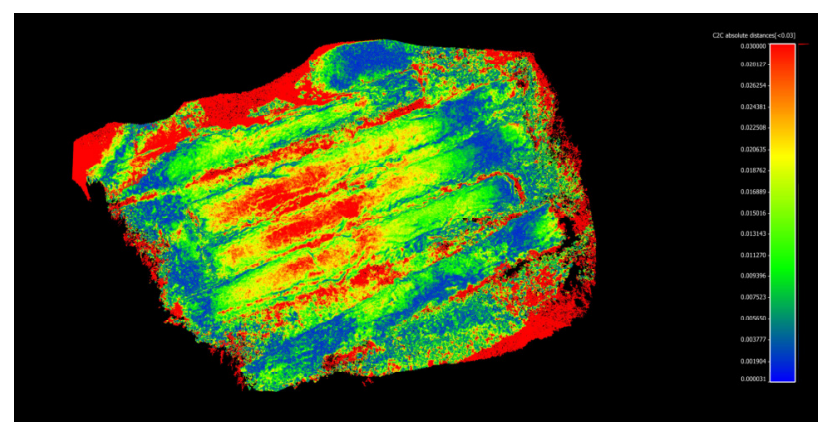

Figure 12. Point clouds of GoPro video photogrammetric survey. Compared distance with points cloud obtained with GoPro images.

\section{CONSIDERATION}

Human activities in the marine environment have been supported -and replaced wherever possible- by remote control equipment that allows to obtain scientific results with less risk to human health. Among the various possibilities currently available, Remote Viewing Vehicles offer not only safety for their operators, but are also a relatively low cost alternative. The employment of a low-cost vehicle adapted to the necessities of surveys support a request for safer, cheaper and more efficient methods for exploring underwater environments, without the high cost of professional ROV, significantly reducing the cost of archaeological operations. On the basis of our experience and some practical tests, still in progress, we can observe that the ROV has some advantages as tool for documentation. Contrary to a diver, it can employ only non-professional cameras that have some metrical limits that can be decreased with the use of special calibrations that are being studied by the IUAV research team.

A second advantage is relative to the use of the ROV in high depths, allowing a first monitoring of the archaeological site and a first survey of the archaeological site, consequently, permitting to reduce the dive time underwater, limiting the physical risks of the divers.

One of the disadvantages is relative to the little dimensions in case of adverse weather conditions; in a situation of heavy current, the OpenROV could hardly maintain the perfect position. The absence of a positioning system, in difficult conditions as open see and great depth, without referencing point of the blue, doesn't permit to reach the bottom on the correct position on the archaeological sites.

\section{REFERENCES}

Bruno, F., Muzzupappa, M., Lagudi, A., Gallo, A., Spadafora, G., Ritacco, G., Angilica, A., Barbieri, L., Di Lecce, N., Saviozzi, G., Laschi, C., Guida, R., Di Stefano, G. 2105. A ROV for supporting the planned maintenance in underwater archaeological sites. Oceans 2015 - Genova, pp.1-7.

Drap, P., Seinturier, J., Hijazi, B., Merad, D., Boi, J-M. 2015. The ROV 3D Project: Deep-Sea Underwater Survey Using Photogrammetry. Applications for Underwater Archaeology. Journal on Computing and Cultural Heritage 8(4), pp. 1-24.

Heisinger, B.E., Holm, W., Fuller, C., -AAIR, Quigg, G.F. 2017. The Hester Lake B-24 Crash: A Case Study for Small, Low-Cost ROVs. doi.org/10.13140/rg.2.2.10440.52488.

Martínez Carvajal, B. V., Sierra Bueno, D. A., Villamizar Mejía, R. 2013. Recent advances in navigation of underwater remotely operated vehicles. Rev. Fac. Ing. Univ. Antioquia N. ${ }^{\circ} 69$, pp. 167-180.

Negahdaripour, S., Firoozfam, P. 2005. An ROV Stereovision System for Ship Hull Inspection. Journal of Oceanic engineering.

Nornes, S.M., Ludvigsen, M., Ødegärd, Ø., Sørensen, A. J. 2015. Underwater Photogrammetric Mapping of an Intact Standing Steel Wreck with ROV. IFAC-PapersOnLine 48-2, pp. 206-211.

Ødegård, Ø., Sørensen, A. J., Hansen, R. E., Ludvigsen, M. 2016. A new method for underwater archaeological surveying using sensors and unmanned platforms. IFAC-PapersOnLine 49-23, pp. 486-493.

Scaradozzi, D., Sorbi, L., Zoppini, F., Gambogi P. 2013. Tools and techniques for underwater archaeological sites documentation. Oceans - San Diego 2013.

Schmidt, V., Rzhanov, Y. 2012. Measurement of microbathymetry with a GOPRO underwater stereo camera pair. Oceans 2012.

Sedlazeck, A., Köser, K., Koch, R. 2009. 3D Reconstruction Based on Underwater Video from ROV Kielk 6000 Considering Underwater Imaging Conditions. - Europe, pp. 110.

Teague, J., Miles, J., Connor, D., Priest, E., Scott, T.B., Naden, J., Nomikou, P. 2017. Exploring offshore hydrothermal venting using low-cost ROV and photogrammetric techniques: a case study from Milos Island, Greece. doi: 10.20944/preprints201710.0014.v1. 